\title{
Congestion Control Mechanisms for 4G Random Access Channel (RACH): A Survey
}

\author{
Magbo P.C., Chukwudi P.C., Abubakar A.
}

\begin{abstract}
Telecommunication systems have evolved from various generations; this evolution aimed at improving the rate of information transfer and communication from one place to another. One of the evolutions in communications is the Long-term Evolution Advanced (LTE-A), also known as the fourth-generation network (4G). The LTE-Advanced, which is a wireless cellular network, offers tremendous services in terms of providing quality of service, security, and improved mobility when compared to the other generation network that predates it. Machine to Machine communications explores the LTE-Advanced technology to supports the massive connection of devices with little or no human interaction. However, one of the challenging issues of Machine-to-Machine communication is congestion. Congestion limits the access of these multiple devices to the network and reduces their response time. This paper presents a survey of different mechanisms that various researchers have proposed over the years to solve the issue of congestion experienced when multiple devices want to have access to the base station (also known as eNodeB).
\end{abstract}

Index Terms - Random Access Channel (RACH), Machine-Typed Communication (MTC), Machine-to-Machine Communication (M2M), Huma-to-Human Communication (H2H) Access Class Barring (ACB), SmRED.

\section{INTRODUCTION}

There are several means of improving the effectiveness of the 3GPP LTE-Advanced network. This paper gives a survey in the contributions of researches in controlling congestion in the $4 \mathrm{G}$ random access channel.

There is widespread of the internet of things (IoT) and global acceptance of the technology as a driving force in interconnecting objects to other objects, people, and systems in significant ways that will benefit society at large. Machine-to-Machine communication is one of the leading frameworks for IoT environments as it offers numerous services and applications. Machine-to-Machine (M2M) communication, also called Machine-type communication by the Third Generation Partnership Project (3GPP), is a technology that involves direct communication between devices. This technology model can be employed when information needs to be transferred between devices with few or no human intervention in the process [1]. The devices referred to in this process can be either the domestic appliances or devices used for designated functions like the sensors and actuators. Machine-to-Machine communication is mostly a hardware-based technology that allows information interaction between machines independently.

If a massive Machine-to-Machine device launches a request on the network simultaneously under one eNodeB (base station), there will be many conflicts leading to congestion since some devices have already gotten preambles. For this reason, important research on how to solve the issue of massive Machine-to-Machine communication traffic overload or congestion in the LTE-Advanced system.

Some reports by $3 \mathrm{GPP}$ have shown that three reasons can be attributed to the signalling congestion experienced during to Machine-to-Machine communication [2]:

1. Machine-to-Machine applications or server(s) active multiple Machine-to-Machine devices simultaneously.

2. Machine-to-Machine devices send many requests in a short period, such as connection, activate, and modification requests.

3. Some applications periodic upload context-aware data, and situations were various sensors are activated at specific times.

Although the data request from these devices are small when a large number of machine-type devices request access over the same channel, they contend to use a shared radio channel and create network overload problems. Handling the massive number of connections generated by a large number of user equipment is an issue that has become a topic of discussion today. Hence, congestion on this network is a factor of a huge number of access requests sent by devices to a single base station during the Random-Access Channel Procedure (RACH procedure).

\section{OVERVIEW OF 4G RANDOM ACCESS CHANNEL (RACH)}

Long Term Evolution Advanced network architecture was formulated by the $3^{\text {rd }}$ Generation Partnership Project (3GPP) in 2004 as a road map for the next generation network that will provide broadband services and connectivity to numerous users [3]. The Third Generation Partnership Project [3], an organization that is responsible for the LTE-A specification, works to identify and propose solutions for the problems and requirements that may arise with the integration of Machine-to-Machine devices into the LTE-A network (4G network). The 4G LTE is a networking standard that presents advantages like mobility, accessibility, good coverage area, security, and other relevant key features for Machine-to-Machine services and applications [1]. The LTE-A networking was designed primarily for Human-to-Human communication; hence some adaptations need to be done for the network to adapt the Machine-to-Machine (M2M) communication requirements. 


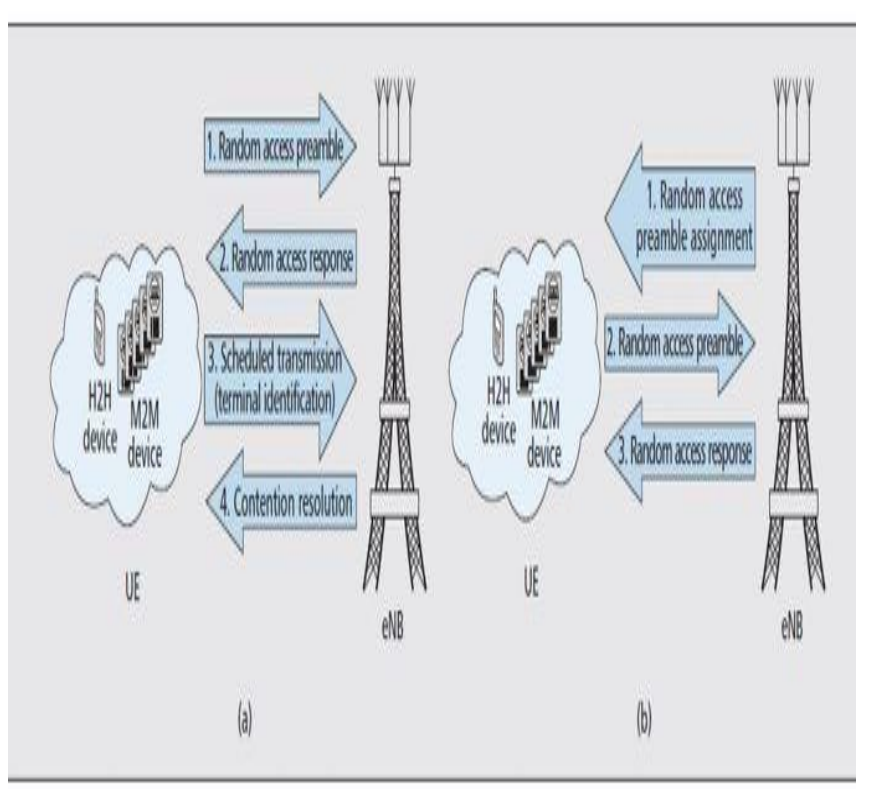

Figure 1: Random Access Procedure in LTE-A; (a) contention-based RA Procedure (b) contention-free RA Procedure

In the 4G network or LTE-Advanced system, a random access (RA) process built on the Random-Access Channel (RACH). The random-access (RA) procedure in the LTE-Advanced system classified into two types: contention-based and contention-free procedures [4]. The user equipment (UE) device normally initiates random access in a contention-based manner by randomly choosing a preamble [4]. A preamble is orthogonal frequency-division multiplexing (OFDM)-based signal using narrower subcarrier spacing. More than one device can choose the same preamble, which necessitates further contention resolution processes. In the case of the contention-free RA, an eNodeB has explicit control over devices to initiate random access by using dedicated preambles. Hence, it is faster than the contention-based approach and mainly used in the handover, where the time delay is crucial.

In LTE-A, a device triggers the contention-based Random Access (RA) procedure to initiate the connection to a base station, also known as eNodeB. In the RA procedure, a device randomly selects a preamble, i.e., a digital signature specified by each eNodeB, and transmits it over the next available RA slot as a connection request to eNodeB. The eNodeB then recognizes the device's access by detecting the preamble signature. In the case of massive M2M devices deployed in one cell, there is a possibility that two or more devices may transmit the same preamble signature over the same RA slot, and thus preamble collisions might be taking place. Consequently, the probability of RA failure rises as the number of preamble collisions increases. Machine-to-Machine applications are very different from classical mobile uses since they present high signalling traffic causing network congestion and overload.

Mechanisms for congestion control in the LTE RAN experienced when massive M2M devices try to establish a connection with the base station (eNodeB) is regarded by 3GPP as a priority issue that needs to be addressed and to enhance Machine type communication over the LTE-Advanced network infrastructure.

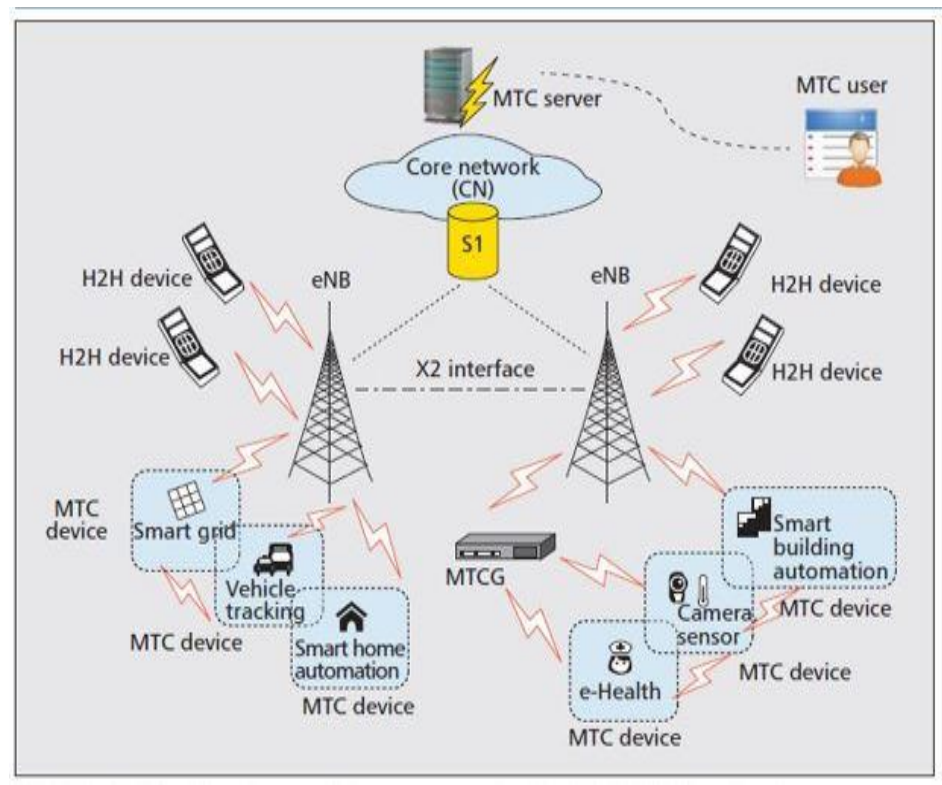

Figure 2: Machine-to-Machine Communication in LTE-A Networks

\section{MECHANISMS FOR CONGESTION CONTROL IN 4G NETWORK}

In [1], the paper proposed a mechanism to control congestion in Long Term Evolution (LTE) network using classes. To accomplish these objectives of reducing congestion problems, they split the Machine-to-Machine devices into high and low priority classes. They then defined the third class for Human-to-Human devices. The results obtained from the research show that their approach can control the impact over Human-to-Human devices, reduce the access delay, and is also compatible with the LTE-A network standard.

In [5], the authors proposed a mechanism to reduce the congestion and overload of RACH by increasing the Random Access (RA) capacity. This can be achieved by increasing the preamble number in the cell, which the devices use to establish a connection with eNodeB. However, increasing the number of preamble numbers in the cell reduces the collision probability, which, by extension, reduces congestion. But this mechanism introduces another challenge whereby the probability of non-orthogonal preambles increases, thereby leading to inter-user interference that can degrade the preamble detection performance. To cope with this new challenge of inter-user interference (IUI), the author proposes the introduction of inter-user interference cancellation (IUIC) architecture to improve the preamble detection process.

A different focus area for controlling congestion was proposed [4]. Here the authors address the congestion challenge from the point where machine type communication devices will select an evolved base node base station (eNodeB) that best provides the quality of service that meets their performance requirements. This approach, which is called the reinforcement learning-based eNodeB selection algorithm, is seen as a good load balancing technique where the machine type communication devices are distributed among the available eNodeB. The Machine type communication devices will have to randomly select the eNodeB that best suits their performance requirement and 
transmit their data with equal probability. With this approach also, eNodeB or the base stations, on the other hand, can allocate resource block $(\mathrm{RB})$ to achieve their desired set objectives. When the resource block allocated by an eNodeB is higher, more Machine type communication devices will choose that eNodeB.

The mechanism of controlling congestion during a random-access channel of the shared medium by reducing the total bytes transmitted was developed by [6]. Transmitting data from numerous Machine type communication devices at the same time will cause traffic and thus degrade the quality of service and response time from the LTE network [7]. This proposal focuses on solving the problem of congestion in both the Random-Access Network (RAN) and the Core Network parts of the LTE network framework. To achieve this, the authors proposed three methods, namely, using a Robust Header Compression (RoHC), eliminating user information, and lightweight compression. In the case of Robust Header Compression, a significant byte of packets was reduced by compressing both the dynamic and static fields in IP header, thereby decreasing the bandwidth usage of the devices and prevent network congestion. In the method of eliminating user information, a coding technique is placed to eliminate the user information within limits defined by the user of the Machine type communication devices. This method will also help to reduce redundant information sent by the Machine type communication device using a programming approach on the device's SIM card. The last method in this control mechanism is the means of lightweight compression. This approach explores the compression that is applied to Machine devices that uses the 4G SIM to transmit data. The compression of the packets originating from the Machine type devices using the 4G SIM will significantly reduce the bandwidth and provide a fast execution time.

The deployment of an Active Queue Management (AQM) strategy as a mechanism for congestion control was proposed in [8]. AQM scheme is used to maintain the average queue size between the upper and lower thresholds of the queue at low oscillations and helps to prevent force drops. To effectively implement a smart congestion control mechanism, the authors used a buffer management scheme called SmRED is introduced and implemented. The SmRED scheme increases the throughput experienced at the low load and reduces the delay at the high load.

An approach that is aimed at controlling congestion for network equipment aside eNodeB was proposed by [9]. The paper adopts the MIH architecture framework which provides a mechanism for optimizing vertical handovers (VHOs) that is independent of the underlying access technologies. The MIH framework developed by IEEE 802.1 workgroup and adopted in this mechanism is an evolution of all networks providing capabilities to detect and initiate handover from one network to another. The congestion control mechanism is divided into three phases, namely; handover preparation, target network selection, and handover decision, and target RAN selection algorithm. This congestion control approach is integrated with the Vertical Handover (VHO) process, and the LTE executes it and the MIH IS, which are key equipment in the whole architecture framework.

Since the preamble is a scarce resource in the LTE-Advanced system, it cannot satisfy all access requests for massive Machine to Machine devices. A focus on the Access Class Barring (ACB) scheme to control congestion in
Machine to Machine communication in real type network system proposed in [10]. Access control is essential to reduce congestion as it principally focuses on controlling the number of access devices where the network system sets a barrier based on some business indicator barring some Machine type communication devices network access. Based on the 3GPP ACB scheme, the paper used a 6D Markov chain to model preamble transfer status and estimate the number of access devices for the next time slot. The whole scheme is called $\mathrm{M}-\mathrm{ACB}$.

The use of a dynamic Access Class Barring (ACB) scheme for congestion control proposed in [11]. The main goal of an ACB scheme as proposed by $3 \mathrm{GPP}$ is to control the arrival of Machine-to-Machine user equipment (UEs) so that the number of transmitted preambles (digital signatures) stays below a certain threshold. This reduces the number of users contending for access at the same time. In this system, an estimate of the current number of Machine-to-Machine devices in the backoff state is used to adjust in real-time the barring rate parameters. The scheme takes a comprehensive view of the access attempts by various devices in the random-access procedure. It then fits the incoming traffic to the RACH capacity by tuning the barring rate appropriately. A device estimator is used to give an estimate of the user equipment in the backoff state and a mechanism for fitting this traffic jointly with the new arrivals in the RACH capacity. An algorithm is then provided for tuning the barring rate dynamically according to the traffic load on the devised estimator. In comparison with the static ACB the dynamic $\mathrm{ACB}$ as proposed in the paper performs better in the sense that increases the probability of successful access while reducing the access delay and the number of preamble transmissions needed to successfully complete the random-access procedure. Also, the dynamic ACB does not affect the human-to-Human $(\mathrm{H} 2 \mathrm{H})$ traffic performance and facilitates the co-existence of $\mathrm{H} 2 \mathrm{H}$ and $\mathrm{M} 2 \mathrm{M}$ traffic.

In [12], a congestion control scheme was proposed to deal with the synchronous correlated arrivals instead of trying to avoid them. The paper proposed the use of a collision resolution algorithm to resolve the synchronous correlated $\mathrm{RACH}$ attempts from the devices. The algorithm work to resolve the synchronous $\mathrm{RACH}$ attempts rather than waste time and LTE resources in trying to avoid them. The basis for the algorithm solution is a q-ary tree splitting technique implemented on top of the existing LTE RACH procedure and activated when RACH overload is detected. The scheme, as proposed in the paper, will allow for the efficient and fast delivery of connection requests from the devices and enabling their processing and inspection by the eNodeB while requiring less amount of resources than the competing schemes. It is expected that this scheme will achieve a reliable and timely massive synchronous access.

A scheme that adopts an algorithm based on adaptive Multiple Access Class Barring (MACB) factors according to the Machine-to-Machine traffic category to overcome the random access overload in 4G RACH was proposed in [13]. The MACB factors consist of two parts; the first is to change the behaviour of the user equipment to allow them to send an indication of congestion for each type of category while the second part is to generate several ACB factors each related to different category. The equipment behaviour can be adapted to three different types of categories namely; the high priority traffic (dedicated to higher emergency alerting), the medium 
priority traffic (dedicated to different $\mathrm{H} 2 \mathrm{H}$ and $\mathrm{M} 2 \mathrm{M}$ like conversational voice and browsing) and the low priority traffic (dedicated to regular monitoring). In the second part, the eNodeB after receiving indicators from the equipment behaviour can estimate the level of congestion and adapt ACB factors for each of the categories and then broadcast those new factors to prevent congestion in the next PRACH slot. Collaborative Distributive Q-learning scheme for addressing the congestion challenge in cellular IoT was proposed in [14]. This mechanism is designed to help the Machine type devices to get unique RA slots for their transmission so that the number of collisions can be reduced. The proposed scheme makes use of the Q-Learning algorithm which used the congestion level of RA slots as a global reward during the learning process. This addresses the RACH congestion problem by taking into consideration the instantaneous cost at the device's end and the global reward function at the network side. The eNodeB computes the global reward and send it to the edge-side through the downlink channel. The calculated instantaneous reward and global information at the edge-side enable devices to update their Q-table and decide the best action at a state. Results have shown that the proposed Q-Learning scheme provides betters performance in terms of convergence time, throughput, and the probability of collision when compared with the existing independent Q-learning approach.

\section{CONCLUSION}

The evolution of telecommunication has significantly improved the rate of information transfer from one source to another within a network infrastructure. In situations where Machine-to-Machine devices communication exists, they can simultaneously launch communication request due to contention for random access channel (RACH). This contention for random access and massive connection of devices in the $4 \mathrm{G}$ RACH has created a new challenge called congestion in the shared medium. The $4 \mathrm{G}$ RACH becomes easily loaded with excessive collisions in the case of massive arrivals of a connection request from the M2M user equipment. This paper has given a comprehensive survey of the impact of congestion and the various control mechanisms for resolving congestions in $4 \mathrm{G}$ Random Access Channel $(\mathrm{RACH})$ as proposed by different researchers.

\section{REFERENCES}

[1] D. Aragao, V. Dario and F. D. C. Miguel, "A Congestion Control Approach for M2M Networks," in The Sixteenth International Conference on Networks, Venice, Italy, 2017.

[2] "System Improvements for Machine-Type Communications," Third Generation Padtnership Project (3GPP), June, 2011.

[3] 3GPP, 3GPP, [Online]. Available: http://www.3gpp.org/about-3gpp/about-3gpp.. [Accessed 17 September 2019].

[4] M. Hassan, H. Ekram and N. Dusit, "Random Access for Machine-to-Machine Communication in LTE-Advanced Networks: Issues and Approaches," IEEE Communications Magazine, pp. 86-93, 2013.

[5] T. Morohashi, L. Chun-Hao, K. Aoi , S. Makoto and M. Hiroyuki , "A High-performance RACH Detection Scheme for Random Access Overload in LTE-Advanced," in IEEE Conference on Standards for Communications and Networking (ICSCN), Tokoyo, Japan, 2015.

[6] J. Joseph, R. Ron and S. Hamman, "Reducing Traffic Congestion for Machine to Machine Type Communication over 4G-LTE Network by Decreasing Total Bytes Transmitted," in 12th IEEE International
Conference on Big Data Science and Engineering, New York, USA 2018.

[7] C.-W. Chang, L. Yi-Hao and C. Jyh-Cheng, "Congestion Control for Machine-Type Communications in LTE-A Networks," in IEEE Gloabl Communications Conference (GLOBECOM), Washington, DC, USA, 2016.

[8] K. Paul, K. Hidehiko, T. Atsuo and H. Teruyuki , "An AQM based congestion control for eNB RLC in 4G/LTE network," in 2016 IEEE Canadian Conference on Electrical and Computer Engineering (CCECE), Vancouver, BC, Canada, 2016.

[9] H. Mzoughi, Z. Faouzi, O. Mohammad S. and K. Lotfi , "3GPP LTE-Advanced Congestion Control Based on MIH Protocol," IEEE Systems Journal, vol. 11, no. 4, pp. 2345-2355, 2017.

[10] J. Liu, Z. Wen'an and S. Lijun, "A Novel Congestion Reduction Scheme for Massive Machine-to-Machine Communication," IEEE Access, vol. 5, pp. 18765 - 18777, 2017.

[11] L. Tello-Oquendo, V. José-Ramón , P. Vicent and G. Luis , "Dynamic Access Class Barring Parameter Tuning in LTE-A Networks with Massive M2M Traffic," in 17th Annual Mediterranean Ad HJoc Networking Workshop, Valencia, Spain, 2018.

[12] G. C. Maduen o, . S. C edomir and P. Petar , "Efficient LTE Access with Collision Resolution for Massive M2M Communications," in 2014 IEEE Globecom Workshops (GC Wkshps), Austin, TX, USA, 2014.

[13] S. Gharbi and Z. Nawel, "Adaptive Multiple Access Class Barring Factors for M2M Communications in LTE-A Networks," in 2015 IEEE 12th Consumer Communications and Networking Conference (CCNC), Nevada, USA, 2015.

[14] S. K. Sharma and W. Xianbin, "Collaborative Distributed Q-Learning for RACH Congestion Minimization in Cellular IoT Networks," IEEE Communications Letters, vol. 23, no. 4, pp. 600-603, 2019.

[15] "System Improvements for Machine-Type Communications," 3GPP, 2011 